\title{
The Promise and Pitfalls of Facebook Advertising: A Genetic Counselor's Perspective
}

Jennifer Verbrugge ${ }^{1}$, Malia Rumbaugh ${ }^{1}$, Lola Cook $^{1}$, Jeanine Schulze ${ }^{1}$, Mandy Miller ${ }^{1}$, Laura Heathers $^{1}$, Vanessa Arnedo ${ }^{2}$, Maggie McGuire Kuhl², Tatiana Foroud ${ }^{1}$

${ }^{1}$ Indiana University School of Medicine, Department of Medical and Molecular Genetics, Indianapolis, IN

${ }^{2}$ The Michael J. Fox Foundation for Parkinson's Research, New York, NY

\section{Correspondence:}

Jennifer L. Verbrugge, MS, CGC, LGC

Department of Medical and Molecular Genetics

Indiana University School of Medicine

410 W. $10^{\text {th }}$ Street

Indianapolis, IN 46202

Tel: 317-278-0298

Fax: $317-278-1100$

Email: jlverbru@iu.edu

This is the author's manuscript of the article published in final edited form as:

Verbrugge, J., Rumbaugh, M., Cook, L., Schulze, J., Miller, M., Heathers, L., ... Foroud, T. (2018). The Promise and Pitfalls of Facebook Advertising: a Genetic Counselor's Perspective. Journal of Genetic Counseling, 27(2), 326-328. https://doi.org/10.1007/s10897-017-0207-3 


\begin{abstract}
Facebook advertising is a powerful tool for increasing the outreach and recruitment of research participants. We describe our experience as genetic counselors within the context of an internet-based research study, recruiting subjects for a Parkinson disease (PD) biomarker study.
\end{abstract}

Key words: social media advertising, Facebook, genetic counseling, Parkinson disease, recruitment

\title{
Facebook Advertising in Genetic Research Recruitment
}

Facebook is the most popular social media platform (SMP) in the United States (Greenwood et al, 2016). Approximately $79 \%$ of online Americans and $68 \%$ of all adults use Facebook, including a growing number of older adult users (62\% of online adults ages 65 and older in 2016 compared to $48 \%$ in 2015$)$. The use of SMPs in research study recruitment has been growing as these tools reach a large number of potential participants in an efficient and cost-effective manner. Facebook recruitment methods utilized in genetics research include paid advertisements, postings, targeted searching and chain referrals (Reaves, A, Bianchi, D, 2013). Paid advertisements, used in several studies, can identify users based on characteristics such as age, geographic region and search history, allowing researchers to focus on participants who potentially meet very specific study criteria. Due to the novelty of using SMPs in research recruitment, recommendations continue to be developed to provide guidance to researchers on how to address concerns surrounding internet recruitment, including ethical issues related to privacy and potential third party tracking (Bender et al., 2017, SACHRP Committee 
Recommendations: Considerations and Recommendations Concerning Internet Research and Human Subjects Research Regulations, with Revisions 2013).

Impact of Facebook Advertisements on the Parkinson's Progression Markers Initiative (PPMI)

\section{Recruitment Process}

The Parkinson's Progression Markers Initiative (PPMI) - a longitudinal, observational biomarkers study - uses web-based recruitment, screening and consent processes (Foroud et al., 2015). PPMI seeks to enroll participants with and without Parkinson disease (PD) who carry mutations in the LRRK2 and/or GBA genes. Given the study's 33 global sites and funds to cover participant travel, its recruitment base is geographically widespread. Participants complete an online screening and consenting process and are eligible for genetic testing if answers to screening questions regarding ethnicity, PD status and family history indicate an increased risk of carrying a $L R R K 2$ or GBA gene mutation. People who have had previous $\angle R R K 2$ and $G B A$ mutation testing may also qualify to participate. All eligible volunteers are contacted by study staff to confirm understanding of the study process and answer any questions before a testing kit is mailed. Participants also have the option to consult with a genetic counselor prior to testing, though most do not choose this option. After testing is complete, genetic counselors provide telephone-based genetic counseling and results disclosure to all participants; those who carry qualifying mutations are then referred to the biomarker phase of the study. PPMI genetics recruitment is coordinated by the study's Genetics Coordination Core at Indiana University. Additional studies are underway to assess participants' satisfaction with the genetic counseling process, as well as the psychological impact of the testing. 
The study sponsor, the Michael J. Fox Foundation for Parkinson's Research (MJFF), first used traditional means of outreach (e.g., mailings, email and in-person events) to send potential participants to the online screening site. This led to the identification of 102 individuals with a LRRK2 mutation in a six-month period (Foroud et al., 2015). To hasten recruitment, MJFF piloted paid Facebook advertisements in the summer and fall of 2015 . While this recruitment approach was fairly novel, the Foundation had digital marketing staff and experience with the platform to leverage. The pilot allowed PPMI to evaluate the cost-benefit relationship of this approach, as well as audience comfort with receiving recruitment messaging via social media. The number of individuals visiting the study website and completing the screening questionnaire and consent immediately increased. The pilot proved a cost-effective strategy, and MJFF did not receive any complaints from Facebook users about the approach.

A more formal and regular Facebook-paid advertising campaign started in January 2016 to identify users more likely to qualify for the PPMI study based on age, geographic area and interests. Following initiation of the campaign, there was a significant increase in participants who consented and qualified for the study. Comparing two three-month periods in 2015 and 2016 (before and after Facebook ads), the number of individuals completing the online consent process more than tripled (257 versus 1200). More importantly, the number of post-genetic counseling sessions increased from 249 to 830. 
This recruitment increase resulted in the need for additional genetic counselors. Due to a local shortage of genetic counselors, we established the additional positions as remote positions, which was compatible with the design of this study. The genetic counseling team expanded from one full-time genetic counselor in 2014 to 4.3 genetic counselors in 2016; all are full-time employees except for one hourly employee who is also employed elsewhere.

As of April 2017, we have counseled over 5,000 participants with positive or negative mutation results. These post-test sessions include results disclosure, pedigree intake, risk assessment and referral to the biomarker phase for participants with mutation positive results. We developed additional strategies to meet the needs of the expanding participant volume and increase efficiency of the genetic counseling process. Participants are now encouraged, though not required, to view an educational video prior to the counseling session, which is emailed via hyperlink (https://youtu.be/hMCRiRH5i4E) at the time of scheduling. Session paperwork and documentation were also streamlined, which include a letter summarizing test results, test reports, and a PD fact sheet-mailed to participants following the genetic counseling session. The introduction of remote workers increased the demand for off-site technical support, and importantly, all remote communication had to comply with Indiana University's HIPAA (Health Insurance Portability and Accountability Act) requirements. Session paperwork generated by remote staff was added to a shared drive, which was limited to designated users who logged in using a secure, university-regulated username and password.

\section{Practice Implications and Conclusions}


Our experience in this research setting illustrates that SMP advertising may lead to rapidly expanding participant volume. This change in workload may require careful analysis of work flow and implementation of strategies to improve efficiency. We also show that a research study utilizing SMP may need to hire additional genetic counselors to meet the demand, and consideration of remote staff may be an appropriate option. This may represent nontraditional job opportunities for genetic counselors that may be either short or long-term, part- or fulltime. It is important to note that telephone genetic counseling services can require multiple state licenses, which may be a barrier to a smaller study due to cost and time involved. Our team opted to "divide and conquer" those states requiring licensure, with each counselor being assigned a specific set of states. However, even with this strategy, this has represented a major logistic and financial burden.

In summary, Facebook advertising was very successful in increasing recruitment of qualified participants to our research study. This increase quickly led to modifications in study workflow, requiring the hiring of multiple remote genetic counselors and support staff. Our experience demonstrates that more non-traditional service delivery modes may help to meet genetic counseling needs - especially as genetic clinics and studies look to innovation and new technologies. Furthermore, additional research is needed to explore not just the impact of SMPs on the study workflow as it relates to genetic counselors, but also on the recruited participants.

\section{Acknowledgements}


We thank the participants and their families for their support of this research study. PPMI - a public-private partnership - is funded by The Michael J. Fox Foundation for Parkinson's Research and funding partners, including Abbvie, Avid Radiopharmaceuticals, Biogen, Bristol Myers Squibb, GE Healthcare, BioLegend, Genentech, GlaxoSmithKline, Eli Lilly and Company, Lundbeck, Merck, Meso Scale Discovery, Pfizer, Piramal, Roche, Sanofi Genzyme, Servier, Takeda, Teva, and UCB.

Conflict of interest: Jennifer Verbrugge, Malia Rumbaugh, Lola Cook, Jeanine Schulze, Mandy Miller, Laura Heathers, Vanessa Arnedo, Maggie McGuire Kuhl and Tatiana Foroud declare that they have no conflict of interest.

All procedures followed were in accordance with the ethical standards of the responsible committee on human experimentation (institutional and national) and with the Helsinki Declaration of 1975, as revised in 2000 (5). Informed consent was obtained from all patients for being included in the study.

\section{References}

Bender, J. L., Cyr, A. B., Arbuckle, L., \& Ferris, L. E. (2017). Ethics and Privacy Implications of Using the Internet and Social Media to Recruit Participants for Health Research: A Privacy-by-Design Framework for Online Recruitment. Journal of Medical Internet Research, 19(4), e104. https://doi.org/10.2196/jmir.7029

Foroud, T., Smith, D., Jackson, J., Verbrugge, J., Halter, C., Wetherill, L., Sims K., Xin W., Arnedo V, Lasch S, Marek K, and the Parkinson's Progression Markers Initiative. (2015). Novel recruitment 
strategy to enrich for LRRK2 mutation carriers. Molecular Genetics \& Genomic Medicine, 3(5), 404-412. https://doi.org/10.1002/mgg3.151

Greenwood, S., Perrin, A, \& Duggan, M. (2016, November 11). Social media update 2016. Retrieved August 31, 2017, from http://www.pewinternet.org/2016/11/11/social-media-update-2016/

Reaves, A. C., \& Bianchi, D. W. (2013). The role of social networking sites in medical genetics research. American Journal of Medical Genetics. Part A, 161A(5), 951-957. https://doi.org/10.1002/ajmg.a.35903

SACHRP Committee Recommendations: Considerations and Recommendations Concerning Internet Research and Human Subjects Research Regulations, with Revisions. (2013). Retrieved August 31, 2017, from https://www.hhs.gov/ohrp/sachrp-committee/recommendations/sachrprecommendations/index.html 